\title{
A new consistent hybrid algorithm for solution of the PDF equations of turbulent reactive flow
}

Reza Mokhtarpoor, Shabrina Virta Inmas, and Metin Muradoglu

Citation: AIP Conference Proceedings 1558, 322 (2013); doi: 10.1063/1.4825488

View online: $\mathrm{http} / / / \mathrm{dx}$.doi.org/10.1063/1.4825488

View Table of Contents: http://aip.scitation.org/toc/apc/1558/1

Published by the American Institute of Physics 


\title{
A New Consistent Hybrid Algorithm for Solution of the PDF Equations of Turbulent Reactive Flow
}

\author{
Reza Mokhtarpoor, Shabrina Virta Inmas and Metin Muradoglu \\ Department of Mechanical Engineering, Koc University, Rumelifeneri Yolu, Sariyer, 34450, Istanbul, Turkey
}

\begin{abstract}
This paper presents a newly developed consistent hybrid finite-volume (FV)/particle algorithm for solution of joint PDF (JPDF) model equation of turbulent reacting flows. In this approach, the open source FV package of OpenFOAM is employed to solve the Favre-averaged mean mass and momentum equations using pressure-based PISO algorithm while a particle-based Monte Carlo algorithm is used to solve the fluctuating velocity-turbulence frequency-compositions JPDF transport equation. In the earlier hybrid method [2,3], a density-based FV algorithm was used to solve the mean flow equations but it has been found to be too dissipative and yet not very robust for incompressible or nearly incompressible flows mainly due to stiffness of the compressible flow equations in the low Mach number limit. In the this work, the density-based FV algorithm is first replaced with a pressure-based PISO algorithm to tackle this problem and then applied for simulation of the Sydney swirl stabilized bluff-body flame SM1. All the equations solved by the FV and particle algorithms are directly derived from the modeled JPDF transport equation so the present method is completely consistent at the level of governing equations. The position and velocity correction algorithms [3] are used to enforce full constancy at the numerical solution level. The results are found to be in a good agreement with the available experimental data and the recent computational results of De Meester et al. [1].
\end{abstract}

Keywords: hybrid method, JPDF, turbulent reactive flow, OpenFOAM

PACS: $47.27 . \mathrm{Ei}, 47.70 . \mathrm{Pq}$

\section{INTRODUCTION}

Turbulent reactive flow retains its vitality in engineering science as an important subject both in the theory and application. Most of important global issues such as energy efficiency, climate change, and pollution are related to the conversion of chemical energy to heat energy via a combustion processes that occur in turbulent flow.

Over the past 20 years computational solution for turbulent combustion have improved dramatically spurred by major advances in physical models, numerical solution algorithms, and most importantly computer power. Probability density function (PDF) methods have emerged as one of the most powerful approaches for accommodating the effects of turbulent fluctuations in velocity and/or chemical composition in CFD modeling of turbulent combustion. Compared to conventional moment closure approaches, the PDF methods offer distinct advantages of being able to treat the important processes of convection and non-linear chemical reaction exactly without any assumptions [10]. In particular, the exact treatment of chemical reactions makes the PDF approaches very attractive for modeling of turbulent combustion. However, an efficient numerical solution algorithm is of crucial importance for application of PDF method for modeling of turbulent reacting flows of practical interest.

The consistent hybrid method developed by Muradoglu et al. [2,3] has proven to be an efficient way of solving the modeled JPDF evolution equation and is also used in the present study. This method combines the best features of the finite volume and particle-based Monte Carlo methods and avoids their respective deficiencies. In the original hybrid method [2,3], a density-based FV algorithm is used to solve the Favre-averaged mean conservation equations for mass, momentum and energy while a particle-based Monte Carlo method is used to solve the modeled PDF transport equation for the fluctuating velocity, turbulent frequency and compositions. Although the density-based FV method is suitable for solving compressible flow equations, it is found to be too dissipative and yet not very robust for incompressible or nearly incompressible flows mainly due to stiffness of the compressible flow equations in the low Mach number limit.

In the present work, the density-based FV solver in the original hybrid method is replaced by a pressure-based FV solver that utilizes the PISO algorithm [12]. For this purpose, the particle algorithm is combined with the OpenFOAM [8], the open source FV package that is freely available from the internet. In the new hybrid algorithm, the FV and particle codes are coupled to form a complete solution algorithm as follows: The mean velocity and mean pressure fields are supplied to the particle code by the FV solver which in turn gets all the Reynolds stresses and mean density fields from the particle code. It is emphasized here that the present hybrid algorithm is completely consistent at the 
level of governing equations solved by the particle and FV algorithms. In addition, the correction algorithms developed by Muradoglu et al.[3] are used to enforce full consistency at the numerical solution level. Although not included in this paper, the present hybrid method has been first validated for the non-swirling inert and reacting bluff-body flows. It is found that the new hybrid algorithm is very robust and the results compare well with the earlier PDF simulations of the same flows reported by Muradoglu et al. [7]. In this work the new hybrid algorithm is employed to simulate the Sydney swirling bluff-body flame SM1. In computations, the simplified Langevin model (SLM) for velocity, the modified Jayesh-Pope model (JPM) for the turbulent frequency and IEM model for the mixing are employed. A simple flamelet chemistry model is used to facilitate extensive numerical simulations. Computational results are well compared with experiment [4, 6,9] and recent joint composition PDF modeling of De Meester et al. [1].

\section{JOINT PDF MODELING}

In the JPDF method, a turbulent flow is modeled through the one-point, one-time joint PDF of selected flow properties. The state of the flow at any location $\mathbf{x}$ and time $t$ is fully described by the three components of velocity $\mathbf{U}=$ $\left(U_{1}, U_{2}, U_{3}\right)$ and by a set of $n_{\phi}$ scalars $\Phi=\left(\phi_{1}, \phi_{2}, \phi_{3}, \ldots, \phi_{n_{\phi}}\right)$ which are the mass fractions of the $n_{s}=n_{\phi}-1$ species and the enthalpy and also by turbulent frequency $\omega$. The velocity-turbulent frequency-compositions JPDF model provides a complete closure for turbulent reacting flows [10]. At position $\mathbf{x}$ and time $t$, the mass-weighted joint PDF $\tilde{f}(\mathbf{V}, \Psi, \theta ; \mathbf{x}, t)$ is defined as

$$
\langle\rho\rangle \tilde{f}(\mathbf{V}, \Psi, \theta ; \mathbf{x}, t) \equiv \rho(\Psi)\langle\delta(\mathbf{V}-\mathbf{U}) \delta(\Psi-\Phi) \delta(\theta-\omega)\rangle,
$$

here angle bracket, \langle\rangle , denotes the Reynolds average, $\rho$ is the density determined as the function of compositions, and $\mathbf{V}, \Psi$, and $\theta$ are independent sample space variables for $\mathbf{U}, \Phi$ and $\omega$, respectively. Two basic properties of $\tilde{f}$ are

$$
\begin{array}{r}
\int \tilde{f}(\mathbf{V}, \Psi, \theta ; \mathbf{x}, t) d \mathbf{V} d \Psi d \theta=1, \\
\tilde{Q}=\int Q(\mathbf{V}, \Psi, \theta ; \mathbf{x}, t) \tilde{f}(\mathbf{V}, \Psi, \theta ; \mathbf{x}, t) d \mathbf{V} d \Psi d \theta
\end{array}
$$

here $\tilde{Q}$ is the Favre-averaged mean of $Q$ which is any random function of velocity $\mathbf{U}$, compositions $\Phi$, and $\omega$. In the present PDF approach, the exact transport equation for $\tilde{f}$ is derived from the mass, momentum and compositions conservation equations and the unclosed terms are modeled through construction of stochastic differential equations (SDEs) governing selected flow properties such that the implied JPDF by SDEs is equivalent to the modeled JPDF as described by Pope $[10,11]$.

\section{Numerical Algorithm}

The particle Monte Carlo technique is the main tool to solve the JPDF equation due to its high dimensionality. In this method the flow is represented by set of notional particles. Stochastic differential equations are then constructed to model the evolution of all particle properties (e.g., particle velocity) so that a stochastically equivalent system is formed in a way that particles exhibit the same JPDF as given by modeled JPDF transport equation.

For the solution of these SDEs the hybrid FV/particle method is employed. In the hybrid approach, FV method is used to solve mean conservation equations. In the present hybrid approach, pressure-based FV solver of OpenFOAM is utilized to solve the the mean conservation equations for mass and momentum, to obtain Favre-averaged velocity. In the particle method, the transport equation for the modeled joint PDF of fluctuating velocity, turbulent frequency, and compositions is derived from the modeled joint velocity-turbulent frequency-compositions PDF transport equation and is solved by a particle-based Monte Carlo algorithm. The FV and particle algorithm are then coupled by exchanging the mean fields required to close the mean conservation and particle evolution equations.

\section{RESULTS}

The new hybrid method has been first validated for the non-swirling isothermal and reacting bluff-body flows. It is found that the new algorithm is very robust and results compare well with the earlier PDF simulations of the same 
flows reported by Muradoglu et al. [7]. In this work the algorithm is employed to simulate more complex flame, the Sydney swirling bluff-body flame SM1. A schematic of the Sydney swirl burner configuration used in this work is given in Figure 1(left). It has a $50 \mathrm{~mm}$ diameter bluff-body $\left(D_{b}=50 \mathrm{~mm}\right)$ with a $3.6 \mathrm{~mm}$ central fuel jet. Swirling air is provided through a $60 \mathrm{~mm}$ diameter annulus surrounding the bluff-body. The burner is placed inside a wind tunnel with square cross section. A wide range of experiments has been performed on this flame $[4,5,6]$. These test cases are distinguished by five independent parameters: the bulk axial velocity of the central jet $\left(U_{j}\right)$, the bulk axial and tangential velocities of the swirling air annulus $\left(U_{s}\right.$ and $\left.W_{s}\right)$, the bulk axial velocity of the coflow of the wind tunnel $\left(U_{e}\right)$ and also with the type of fuel. We considered the case SM1 for which the flow parameters are summarized in Table 1 . Here $S_{g}$ is geometric swirl number defined as $S_{g}=W_{s} / U_{s}$.

TABLE 1. Flow parameters of the case SM1

\begin{tabular}{ccccccc}
\hline case & fuel & $U_{e}(m / s)$ & $U_{j}(m / s)$ & $U_{s}(m / s)$ & $W_{s}(m / s)$ & $S_{g}$ \\
\hline SM1 & CH4 & 20 & 32.7 & 38.2 & 19.1 & 0.5
\end{tabular}

For axisymmetric simulation of this flow a cylindrical coordinate system $(x, r, \theta)$ has been adopted. The origin of the coordinate system is on the centerline of the jet exit plane $(x=0, r=0)$. Here $x$ and $r$ represents the axial and radial directions, respectively. The computational domain which is used for calculations is a rectangular domain with $0.5 \mathrm{~m}$ $\left(10 D_{b}\right)$ in the axial direction downstream of bluff-body and $0.2 \mathrm{~m}\left(4 D_{b}\right)$ in the radial direction, where $D_{b}=50 \mathrm{~mm}$ is the diameter of bluff-body. For boundary conditions, the mean axial velocity is specified by fully-developed turbulent pipe flow. Experimental data is used in the primary swirling air stream for axial and tangential velocities and also for axial velocity in coflow region. Slip boundary condition is applied in bluff-body wall. Extensive computations are performed to show the performance of the JPDF method and hybrid solution algorithm. The statistical stationarity and grid convergence are first examined and it is found that a statistically stationary solution is reached after about 10000 particle time steps and grid independent solutions are obtained with a Cartesian non-uniform grid.
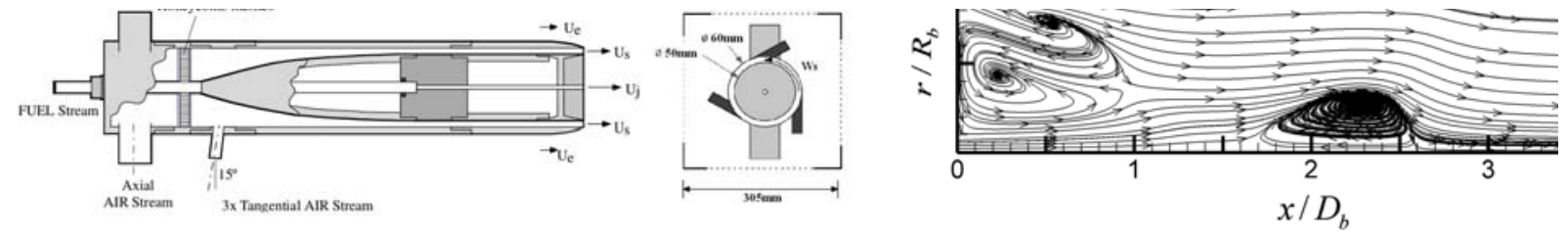

FIGURE 1. Schematic drawing of the Sydney flame (left) and the computed streamlines in the downstream of the burner exit (right).

Flow feature for the flame SM1 is typically of a swirl flow past a bluff-body. As is shown in Figure 1(right), due to the flow separation from circular bluff body, there is a toroidial recirculation zone (RZ) immediately behind the bluffbody base and further downstream there is a vortex breakdown bubble (VBB) around the centerline due to the swirl effect as also observed in the experiment [4]. The upstream RZ has two vortices which center of the first is located in $x / D_{b} \simeq 0.25$ and the second one is in $x / D_{b} \simeq 0.5$ that extends up to $x / D_{b} \simeq 0.9$. The VBB is located between $x / D_{b} \simeq 1.8$ and $x / D_{b} \simeq 2.5$. Experimental observations reported the centre of the second RZ at $x / D_{b} \simeq 1.7$ which extends up to $x / D_{b} \simeq 2.2$ [5]. Figure 2 shows the computed radial profiles of mean axial and tangential velocities, rms fluctuating axial velocities, mean mixture fraction, and rms mixture fraction at various axial locations together with the experimental data and the compositions PDF simulation results of De Meester et al. [1]. There are some quantitative differences between our results and previous computed profiles, which can be attributed to the use of different PDF approaches and chemistry models. Qualitatively both computed profiles are similar to experimental profiles. Except in the the axial location of $x / D_{b}=0.4$ near the axis of symmetry which calculated results have major differences with experimental data the results are in good agreement with measurements and also the results of De Meester et al. In conclusion, the present hybrid method is found to be a lot more robust than the hybrid algorithm developed by Muradoglu et al. $[2,3]$ while retaining all the advantages of the consistent hybrid approach.

\section{ACKNOWLEDGMENTS}

This work is supported by the Scientific and Technical Research Council of Turkey (TUBITAK), Grant No.111M067. 

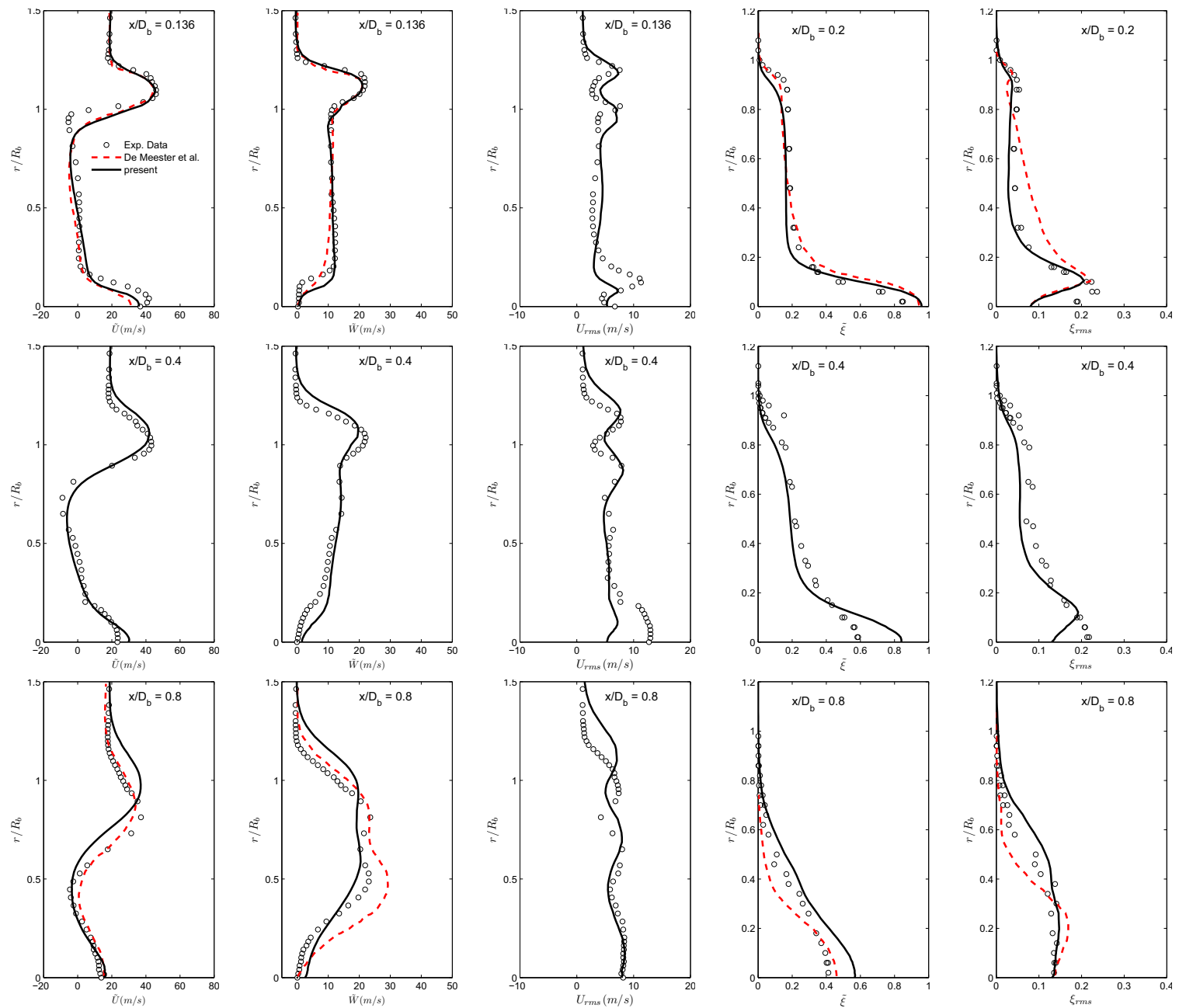

FIGURE 2. Profiles of mean and rms quantities compared with experimental data [9] and numerical results of De Meester et al. [1].

\section{REFERENCES}

1. R. De Meester, B. Naud , U. Maas, and B. Merci, Combust. Flame 159:2415-2429 (2012).

2. M. Muradoglu, P. Jenny, S.B. Pope, and D.A. Caughey, J. Comp. Phys. 154:342-371 (1999).

3. M. Muradoglu, S.B. Pope, and D.A. Caughey, J. Comp. Phys. 172:841-878 (2001).

4. Y.M. Al-Abdeli, and A.R. Masri, Combust. Theory Model 7:731-766 (2003).

5. P.A.M. Kalt, Y.M. Al-Abdeli, A.R. Masri, and R.S. Barlow, Proc. of the Combust. Inst. 29:1913-1917 (2002).

6. A.R. Masri, P.A.M. Kalt, and R.S. Barlow, Combust. Flame 137:1-37 (2004).

7. M. Muradoglu, K. Liu and S.B. Pope, Combust. Flame 132:115-137 (2003).

8. OpenFOAM, The open source CFD toolbox, OpenFOAM, website http://www.openfoam.com/ (2013).

9. Univ.of Sydney, Clean Combust. Research Group http://sydney.edu.au/engineering/aeromech/thermofluids/swirl.htm (2013).

10. S.B. Pope, Prog. Energy Combust Sci. 11:119-192 (1985).

11. S.B. Pope, Ann. Rev. Fluid Mech. 26:23-63 (1994).

12. R. I., Issa, J. Comp. Phys. 62:40-65 (1986). 